\title{
Reduced Priority MELD Score for Hepatocellular Carcinoma Does Not Adversely Impact Candidate Survival Awaiting Liver Transplantation
}

\author{
P. Sharma ${ }^{a}$ * , A. M. Harper ${ }^{b}$, J. L. Hernandez ${ }^{c}$, \\ T. Heffron ${ }^{d}$, D. C. Mulligan ${ }^{\mathrm{e}}$, R. H. Wiesner ${ }^{f}$ \\ and V. Balan' \\ a Division of Gastroenterology, University of Michigan, \\ Ann Arbor, Michigan, USA \\ b United Network for Organ Sharing/Organ Procurement \\ and Transplant Network, Richmond, Virginia, USA \\ ${ }^{c}$ Division of Biostatistics, Mayo Clinic, Scottsdale, \\ Arizona, USA \\ 'Department of Surgery, Emory University Hospital, \\ Atlanta, Georgia, USA \\ e Division of Transplantation Surgery, Mayo Clinic, \\ Scottsdale, Arizona, USA \\ ${ }^{\dagger}$ Division of Gastroenterology and Hepatology, Mayo \\ Clinic, Rochester, Minnesota, USA \\ 'Division of Transplantation Medicine, Mayo Clinic, \\ Scottsdale, Arizona, USA \\ * Corresponding author: Pratima Sharma \\ pratimas@med.umich.edu
}

The liver organ allocation policy of the United Network for Organ Sharing (UNOS) is based on the model for end-stage liver disease (MELD). The policy provides additional priority for candidates with hepatocellular carcinoma (HCC) who are awaiting deceased donor liver transplantation (DDLT). However, this priority was reduced on February 27, 2003 to a MELD of 20 for stage T1 and of 24 for stage T2 HCC. The aim of this study was to determine the impact of reduced priority on HCC candidate survival while on the waiting list. The UNOS database was reviewed for all HCC candidates listed after February 27, 2002, The HCC candidates were grouped into two time periods: MELD 1 (listed between February 27, 2002, and February 26, 2003) and MELD 2 (listed between February 27, 2003 and February 26, 2004). For the two time periods, the national DDLT incidence rates for HCC patients were 1.44 versus 1.53 DDLT per person-year $(p=N S)$ and the waiting times were similar for the two periods $(138.0 \pm 196.8$ vs. $129.0 \pm 133.8$ days; $p=$ NS). Furthermore, the 3-, 6and 12-month candidate, patient survival and dropout rates were also similar nationally. Regional differences in rates of DDLT for HCC were observed during both MELD periods. Consequently, the reduced MELD score for stage T1 and T2 HCC candidates awaiting DDLT has not had an impact nationally either on their survival on the waiting list or on their ability to obtain a liver transplant within a reasonable time frame. However, regional variations point to the need for reform in how organs are allocated for $\mathrm{HCC}$ at the regional level.

Key words: Deceased donor liver transplantation, hepatocellular carcinoma, model for end-stage liver disease

Received 27 September 2005, revised 17 April 2006 and accepted for publication 24 April 2006

\section{Introduction}

Liver transplantation (LT) remains the only curative option for majority of patients of early stage hepatocellular carcinoma (HCC) and offers the best chance for cure and long-term survival $(1,2)$. The candidates at risk for HCC include those with cirrhosis associated with hepatitis $B$ and $\mathrm{C}$, and certain metabolic disorders like hemochromatosis and alpha-1-antitrypsin deficiency. Most HCC develop in cirrhotic livers, where surgical resection is not a viable option because the functional hepatic synthetic reserve is not sufficient to tolerate the removal of hepatic parenchyma (3).

The conventional Milan's criteria that predict low risk for HCC recurrence after LT came from a prospective study by Mazzaferro et al. from Milan, Italy (1). Several other reports have supported the finding that LT for early stage HCC achieves excellent long-term survival rates that are equal to or superior to those for patients with nonmalignant indications $(1,2)$

The new liver allocation policy, based on model for endstage liver diseases (MELD), assigns priority to waiting candidates on the basis of their risk of death within 3 months (4). The original MELD-based allocation plan estimated a $15 \%$ risk (MELD score 24 ) and a $30 \%$ risk (MELD score 29) of disease progression beyond stage T1 and $T 2$, respectively, in accordance with the classification adapted by United Network for Organ sharing (UNOS) from American-modified Tumor-Node-Metastasis Staging Classification (5). UNOS defines stage T1 as 1 nodule of 1.9 $\mathrm{cm}$ or less and stage T2 as 1 nodule of $2-5 \mathrm{~cm}$ or as 2 or 3 nodules all larger than $3 \mathrm{~cm}(5)$

In a previous report by our group that examined the UNOS database for the impact of this new allocation policy on 
HCC candidates, we found that significantly higher proportions of candidates listed with a diagnosis of HCC were receiving deceased donor liver transplantation (DDLT) (6). Both the dropout rate and death in the absence of transplantation were significantly lower in HCC patients.

The allocation policy was therefore amended and implemented on February 27, 2003. For stage T1 HCC, the MELD score was reduced from 24 to 20, and for stage T2, the MELD score was reduced from 29 to 24 . The MELD scores of 24 and 20 correspond respectively to a $15 \%$ and $8 \%$ risk of becoming ineligible for transplantation and dropping off the list because of disease progression in a 3-month period (5). The aim of this study was to assess the impact on HCC patients of the reduction in priority of MELD scores.

\section{Methods}

Data from the UNOS database for LT were collected for all adult HCC candidates who had registered with UNOS as a LT candidate or recipient or who had an exceptional case request and listed after February 27, 2002 corresponding to the implementation of the new MELD allocation plan. Patients were grouped by two time periods. MELD 1 referred to the candidates listed between February 27, 2002 and February 26, 2003, corresponding to the time period when MELD scores of 24 and 29 were assigned for stage $\mathrm{T} 1$, and T2 HCC, respectively. MELD 2 referred to the candidates listed for HCC between February 27, 2003 and February 26, 2004. This time frame corresponded with the period when MELD scores were reduced to 20 from 24 for stage T1 and from 29 to 24 for stage T2 HCC. The organ procurement transplant network captures waiting list additions, modifications and removal from the member transplant centers in real time through $\mathrm{UNet}{ }^{\mathrm{sm}}$, an Internet-based data collection system. Demographic information and clinical data on candidates and recipients are entered using online forms. The following data were collected: age and gender at listing, date of listing, date of DDLT, serum bilirubin, creatinine and international normalized ratio for prothrombin time at listing and at LT. In addition, the following data were also collected for the two time periods: candidate's status on the list (including those who remained on the waitlist in an inactive status due to various reasons), dropoff from the waitlist due to death or too sick to be transplanted, date and reason for removal from the list, survival on the list and after DDLT.

DDLT incidence rates, waiting time, candidate survival while on the waiting list, candidates removed from the waiting list (dropout rate) and patient survival after DDLT were compared for both periods. The time spent by the candidates on the waiting list was used to calculate the person-years in both time periods. The DDLT incidence rates were obtained by dividing the total number of transplant with person-years and then expressing them as DDLT per person-year. Candidates who died or became too sick to undergo transplantation were removed from the waiting list and included in the calculation of the dropout rate. The inactive candidates that remained on the waiting list in an inactive status were used to calculate the proportion of candidates in inactive status for the two time periods. The inactive candidates were not included in the calculation of the dropout rate. Candidates with living donor liver transplant were excluded from the analysis. MELD scores were calculated as described previously $(4,6)$. Patients whose disease progressed to stage T2 were included in stage T2 category. The time spent classified as stage $T 1$ was counted toward a calculation of the stage $T 1$ person-years and the time spent as stage $\mathrm{T} 2$ was counted toward a calcula- tion of the stage T2 person-years. Some candidates were grandfathered in with a MELD score of 24 or 29 in the MELD 2 period. For these candidates, the time spent on the waiting list during MELD 1 and MELD 2 was counted toward the calculation of the MELD 1 and MELD 2 person-years.

The results for continuous variables were expressed as mean \pm SEM, and qualitative variables were expressed as proportions. As this was an intentto-treat analysis, all the patients were included for the outcomes analysis. The continuous variables were analyzed using the Student's $t$-test and categorical variables were analyzed using the chi-square test. Kaplan-Meier curves were generated for the candidate and patient survival rates. Candidates were censored for transplantation for candidate survival on the list. The incidence rates of the regions were compared with each other within each MELD period. The Poisson test was used to compare the incidence rates regionally. The chi-square analysis was used to analyze the proportions of inactive patients in the two time periods. A p-value of less than 0.05 was considered statistically significant.

\section{Results}

\section{United States national data}

In the United States, the number of new LT candidates listed for HCC in the UNOS database during the MELD 1 and MELD 2 periods was 1049 and 1093, respectively. Table 1 summarizes the demographics of candidates, the calculated MELD at the time of listing and at DDLT, the dropout rates, and the proportions of candidates listed with stage T1 or stage T2 lesions and the proportion of candidates who had transplants for T1 and T2. The waiting time during MELD 1 and MELD 2 was $138.3 \pm 196.8$ and $129.5 \pm 133.8$ days $(p=0.29)$, respectively. The 12 -month candidate survival on the waiting list was $88 \%$ and $89 \%$ $(p=0.44)$ during MELD 1 and 2, respectively.

Of 1049 HCC candidates, 828 (78.9\%) underwent DDLT during MELD 1; in MELD 2, 818 (74.8\%) of 1093 HCC patients underwent DDLT. The total number of person-years was 575.2 for MELD 1 and 532.6 for MELD 2. DDLT incidence rates during MELD 1 and MELD 2 were 1.44 and 1.53 DDLT per person-year $(p=0.19)$. In MELD 2, a significantly smaller proportion of HCC candidates with T2 lesions was listed and received transplants. There was no difference in 3-, 6- and 12-month patient survival after DDLT. The proportion of candidates who remained on the waiting list with an inactive status was similar in both time periods (6.8\% in MELD 1 and $7.5 \%$ in MELD 2; $p=0.5)$.

\section{Regional data}

The impact of the reduced priority for HCC was also assessed in the 11 UNOS regions across the United States. The DDLT incidence rates for HCC during MELD 1 were highest in regions 3 and 10 compared with the rates for the other regions $(p<0.001)$. Also during MELD 1, region 9 had a lower DDLT incidence rate for HCC compared with that of the other regions $(p=0.01)$. However, during MELD 2, regions 3, 6 and 10 had higher DDLT incidence rates for HCC than did the other regions $(p=0.01)$. Figure 1 shows the regional difference in DDLT incidence rates during the 
Table 1: Demographics, waiting time, actual MELD score and proportion of candidate listed and transplanted with T1 and T2 lesions and 3-, 6- and 12-month dropout rates

\begin{tabular}{llll}
\hline Variables & MELD 1 & MELD 2 & p-Value \\
\hline Age at listing (N) at listing (year) & $55.5 \pm 8.4(1049)$ & $56.0 \pm 8.0(1093)$ & 0.13 \\
Age at listing (N) at DDLT (year) & $55.1 \pm 8.2(828)$ & $55.7 \pm 8.1(818)$ & 0.11 \\
Sex (\% male) & $75 \%(85 / 1049)$ & $78 \%(850 / 1093)$ & 0.11 \\
Calculated MELD at listing & $11.9 \pm 5.2(1048)$ & $11.5 \pm 4.9(1092)$ & 0.07 \\
Calculated MELD at DDLT & $13.3 \pm 5.9(828)$ & $13.2 \pm 6.1(818)$ & 0.70 \\
Wait time (days) & $138 \pm 197(828)$ & $130 \pm 134(818)$ & 0.29 \\
T1 Stage & $14.2 \%(149 / 1049)$ & $11.8 \%(130 / 1093)$ & 0.11 \\
T2 Stage & $77 \%(808 / 1049)$ & $71.4 \%(781 / 1093)$ & 0.003 \\
Dropout (3 months) & $3.7 \%(39 / 1049)$ & $3.6 \%(40 / 1093)$ & 0.94 \\
Dropout (6 months) & $5.1 \%(54 / 1049)$ & $5.8 \%(63 / 1093)$ & 0.53 \\
Dropout (12 months) & $6.9 \%(73 / 1049)$ & $8.4 \%(92 / 1093)$ & 0.21 \\
3-Month survival on list & $96 \%$ & $94 \%$ & 0.44 \\
6-Month survival on list & $93 \%$ & $91 \%$ & 0.44 \\
12-Month survival on list & $88 \%$ & $89 \%$ & 0.44 \\
3-Month survival after LT & $94 \%$ & $90 \%$ & 0.31 \\
6-Month survival after LT & $89 \%$ & $84 \%$ & 0.27 \\
12-Month survival after LT & $83 \%$ & $84 \%$ & 0.15 \\
\hline
\end{tabular}

Results are expressed as mean \pm standard error of mean (SEM).

$\mathrm{N}=$ number of subjects in the parenthesis.

Table 2: Incidence Rate of DDLT and waiting time during MELD 1 and 2 in all 11 UNOS regions

\begin{tabular}{|c|c|c|c|c|}
\hline Region & & MELD 1 & MELD 2 & p-Value \\
\hline \multirow[t]{3}{*}{1} & Waiting time (days) & $132.3 \pm 227.9$ & $223.5 \pm 187$ & 0.052 \\
\hline & 12-Month survival post-LT & $92 \%$ & $83 \%$ & 0.33 \\
\hline & 12-Month dropout & $11.7 \%(6 / 51)$ & $12 \%(7 / 58)$ & 0.96 \\
\hline \multirow[t]{3}{*}{2} & Waiting time (days) & $113.7 \pm 185.4$ & $101.2 \pm 142.2$ & 0.6 \\
\hline & 12-Month survival post-LT & $82 \%$ & $84 \%$ & 0.78 \\
\hline & 12-Month dropout & $8.7 \%(11 / 126)$ & $6.8 \%(9 / 133)$ & 0.55 \\
\hline \multirow[t]{3}{*}{3} & Waiting time (days) & $72.3 \pm 142$ & $72.8 \pm 131.3$ & 0.98 \\
\hline & 12-Month survival post-LT & $79 \%$ & $90 \%$ & 0.06 \\
\hline & 12-Month dropout & $1.9 \%(2 / 106)$ & $5.4 \%(6 / 110)$ & 0.09 \\
\hline \multirow[t]{3}{*}{4} & Waiting time (days) & $160.4 \pm 257$ & $117.5 \pm 123$ & 0.18 \\
\hline & 12-Month survival post-LT & $95 \%$ & $86 \%$ & 0.06 \\
\hline & 12-Month dropout & $2.9 \%(3 / 102)$ & $8.5 \%(8 / 94)$ & 0.09 \\
\hline \multirow[t]{3}{*}{5} & Waiting time (days) & $201 \pm 218.2$ & $213.4 \pm 155.3$ & 0.57 \\
\hline & 12-Month survival post-LT & $90 \%$ & $90 \%$ & 0.52 \\
\hline & 12-Month dropout & $8.5 \%(22 / 258)$ & $11.9 \%(29 / 243)$ & 0.21 \\
\hline \multirow[t]{3}{*}{6} & Waiting time (days) & $124.3 \pm 179.3$ & $85.1 \pm 115.4$ & 0.30 \\
\hline & 12-Month survival post-LT & $79 \%$ & $86 \%$ & 0.52 \\
\hline & 12-Month dropout & $3.2 \%(1 / 31)$ & $2.6 \%(1 / 39)$ & 0.87 \\
\hline \multirow[t]{3}{*}{7} & Waiting time (days) & $117.9 \pm 155.3$ & $136.2 \pm 126.3$ & 0.37 \\
\hline & 12-Month survival post-LT & $87 \%$ & $81 \%$ & 0.27 \\
\hline & 12-Month dropout & $1.9 \%(2 / 106)$ & $2.6 \%(3 / 116)$ & 0.73 \\
\hline \multirow[t]{3}{*}{8} & Waiting time (days) & $161.6 \pm 274.1$ & $130.0 \pm 151.2$ & 0.50 \\
\hline & 12-Month survival post-LT & $91 \%$ & $82 \%$ & 0.25 \\
\hline & 12-Month dropout & $1.9 \%(1 / 51)$ & $7.5 \%(4 / 53)$ & 0.18 \\
\hline \multirow[t]{3}{*}{9} & Waiting time (days) & $204.4 \pm 244.8$ & $174.0 \pm 132.5$ & 0.34 \\
\hline & 12-Month survival post-LT & $77 \%$ & $84 \%$ & 0.42 \\
\hline & 12-Month dropout & $14.3 \%(19 / 132)$ & $12.7 \%(17 / 134)$ & 0.68 \\
\hline \multirow[t]{3}{*}{10} & Waiting time (days) & $75 \pm 160.6$ & $61.9 \pm 91.6$ & 0.63 \\
\hline & 12-Month survival post-LT & $81 \%$ & $88 \%$ & 0.42 \\
\hline & 12-Month dropout & $5 \%(2 / 40)$ & $1.6 \%(1 / 61)$ & 0.33 \\
\hline \multirow[t]{3}{*}{11} & Waiting time (days) & $171.3 \pm 240.1$ & $178.6 \pm 167.9$ & 0.86 \\
\hline & 12-Month survival post-LT & $93 \%$ & $87 \%$ & 0.69 \\
\hline & 12-Month dropout & $9.4 \%(5 / 53)$ & $11.1 \%(7 / 63)$ & 0.77 \\
\hline
\end{tabular}




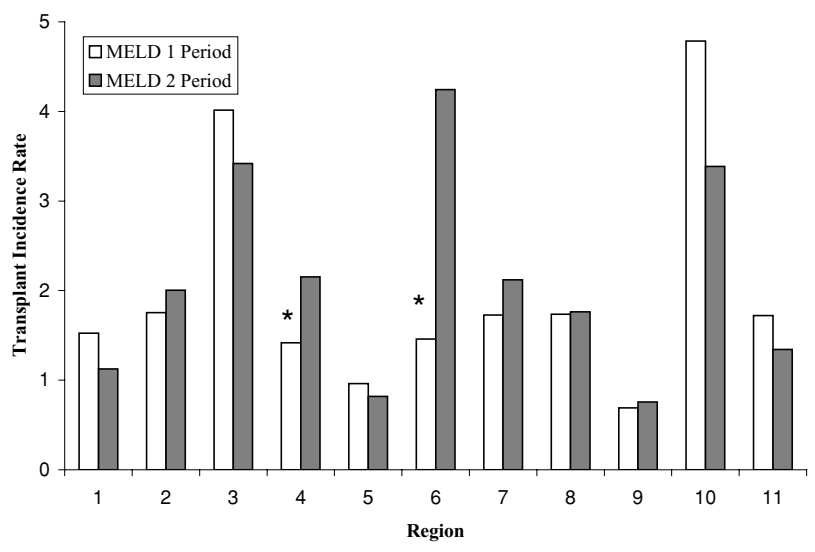

Figure 1: Comparison of incidence rate of DDLT during MELD 1 and 2 time periods in the 11 UNOS regions. *Significant difference between the two MELD periods $(p<0.05)$.

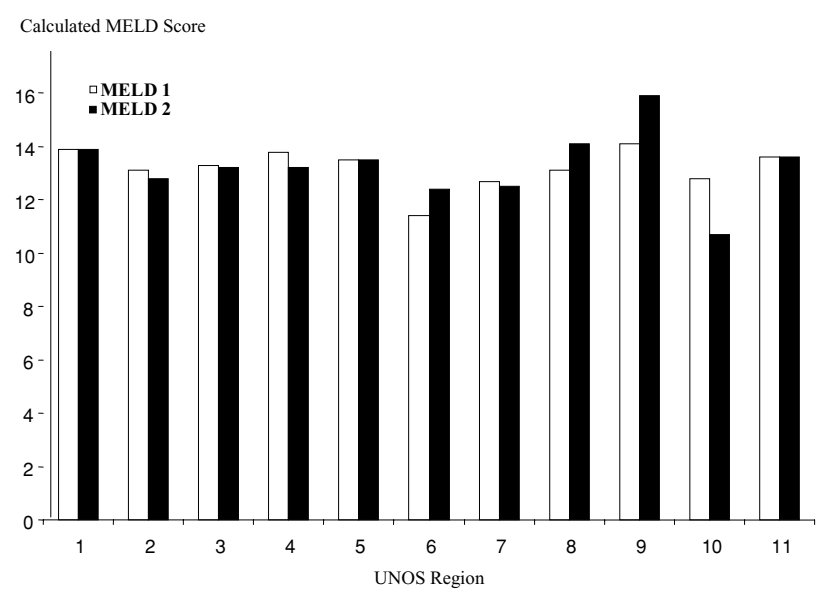

Figure 2: Calculated MELD score during MELD 1 and 2 in 11 UNOS region. $X$-axis shows the region and $Y$-axis shows the MELD score. The calculated MELD score for all HCC candidates in the regions did not change with the change in the $\mathrm{HCC}$ priority MELD.

MELD 1 and MELD 2 periods. Figure 2 demonstrates the calculated MELD score for all HCC candidates. The MELD score in the regions did not increase with the change in the HCC priority MELD score. Therefore, the driving factor for higher MELD scores in the regions has been the exception score for HCC.

The candidates' characteristics, their dropout rates and candidate and graft survival rates were similar in all 11 regions. The waiting time, 12-month dropout rate and 12 -month survival rate after DDLT are summarized in Table 2.

The waiting time varied from region to region. The maximum wait was observed in region 5 . The shortest wait was in regions 3 and 10 . The 12-month dropout rate in either MELD 1 or MELD 2 did not change except in region 4, where there was a trend toward higher dropout in MELD 2 ( $3 \%$ vs. $9 \%$; $p=0.09$ ). In MELD 1, it was $2-5 \%$ in regions $3,4,6,7,8$ and 10 , and it was $9-14 \%$ in regions 1 , 2, 5, 9 and 11. However, in MELD 2, it increased slightly or stayed the same; this change was not statistically significant. Twelve-month survival of patients after LT was similar in both time periods for all regions.

\section{Discussion}

LT is a well-established indication for HCC. Candidates drop off the transplant waiting list because of tumor progression that would make them ineligible for a transplant if the organ is not received within a reasonable period. The MELD-based allocation policy recognized these findings, and HCC candidates were initially given a priority MELD score of 24 for stage T1 HCC and 29 for stage T2 HCC. However, after implementation of this policy, there was concern that HCC candidates were perhaps given excessive priority $(6,7)$. According to national data, $21.7 \%$ of liver transplant candidates with HCC received DDLT after implementation of the MELD-based liver allocation policy, compared with $8.8 \%$ in the pre-MELD era $(6,8)$. When Wiesner et al. examined data from the first 6 months of implementation of the MELD-based allocation policy, they found that $27 \%$ of stage $\mathrm{T} 1 \mathrm{HCC}$ candidates and $45 \%$ of stage T2 candidates received DDLT within 30 days of listing; During the MELD 1 period, more than $87 \%$ of HCC candidates underwent DDLT within 90 days of being listed (9). However, if most stage T2 HCC candidates (87\%) received a liver transplant during the first MELD era within 90 days of being listed, then one might expect the waiting time to be less than 90 days in most regions (9). In our analysis, the national mean waiting time was $138 \pm 197$ days in MELD period 1 and $130 \pm 134$ days in MELD period 2. This disparity is probably due to the fact that we are presenting 1 -year results.

Nevertheless there is still a need for prioritization for patients with stage T2 HCC disease. These patients have $60 \%$ chance of becoming ineligible for a transplant due to disease progression or even dying within 1 year (10). In contrast, patients with stage 1 disease have less than $10 \%$ risk of dying or of becoming ineligible for a transplant within 1 year (10). Accordingly, in February 2003, points allocated to stage T1 and stage T2 HCC were reduced to 20 and 24, respectively, which correspond to an $8 \%$ and $15 \%$ risk, respectively, of dropping off the list. Our study validates this change, which has not affected the T2 HCC patients adversely. We found that the DDLT incidence rates and waiting times for $\mathrm{HCC}$ candidates were similar in both the MELD time periods. Since the waiting list constantly changes, the incidence rates were calculated to correct for changes in size of the waiting list during different times. Nationally, the 12-month HCC candidate 
survival rate, the 12-month dropout rate due to death or deterioration of the candidate's clinical condition and the 1 -year patient survival rate after LT were similar in both periods.

The similar number of patients receiving transplants in MELD 1 and MELD 2 suggests that HCC patients get enough points in both periods to have allocation weighted greatly in their favor. Wiesner et al. reported a transplant rate of $29.4 \%$ and $48.3 \%$ for patients with stage 1 and stage 2 HCC (MELD, 24 and 29, respectively), compared with rates of $16.7 \%(p<0.01)$ and $27.3 \%(p<0.01)$ in candidates with chronic liver disease and a MELD score of 24 or 29 in era 1 (10). A comparison of the DDLT incidence rates for non-HCC patients with comparable calculated MELD scores on the list would be of interest. However, non-HCC candidates with a MELD score of 12-14 have a very low risk of dying while on the waiting list and usually do not receive LT while at that MELD score. Recently, Merion et al. reported that the posttransplantation mortality risk was $3.64,2.35$ and 1.21 times higher than the waitlist mortality risk for MELD scores of 6-11, 1214 and 15-17, respectively (11). Their study excluded HCC candidates

Fewer candidates with stage T2 lesions were listed and received DDLT in the MELD 2 period compared with those in MELD 1. This difference was observed across regions and reflects the positive impact of decreasing the priority of MELD scores for patients with HCC (5). However, there was a trend toward decreased DDLT incidence rates for T1 HCC candidates in MELD 2, perhaps because of the small sample size. The progression of lesions to stage 3 and beyond could not be assessed, because this information is not reported to UNOS. In our opinion, however, such information is crucial and should be reported. Having it available would facilitate the study of the natural progression of tumors using a larger database. Tumor recurrence rates were not calculated because of the shorter follow-up period after LT.

We could not obtain the explant data, however, a singlecenter study found no evidence of $\mathrm{HCC}$ in explants from $33 \%$ of patients who had LT for HCC (12). In a review of pathologic findings for 666 explants UNOS reported for the first 8 months of the MELD system, 94 (14.1\%) had no evidence of $\operatorname{HCC}(10,13)$. In another study, pathologic findings indicated that one-third of patients who met criteria for stage $\mathrm{T} 1$ or stage $\mathrm{T} 2 \mathrm{HCC}$ on imaging studies before LT had disease that had advanced beyond stage 2 , when explant pathology was reviewed. Assessment of the pathologic stage relative to the listing stage, which was based on imaging studies, found that about one-third of the patients were upstaged on the basis of explant pathology. Another major finding was that $31 \%$ of patients undergoing LT for stage T1 HCC lesions had been misdiagnosed during pre-LT assessment and actually had no ev- idence of tumor in the explanted liver (10). This finding reinforces the fact that state-of-the-art imaging studies are not very sensitive in diagnosing stage T1 lesions. In contrast, $91 \%$ of patients with stage T2 HCC had lesions identified by pathologic findings in the explanted liver (10). Our study suggests that the reduction in prioritization of $\mathrm{HCC}$ candidates for LT has not had a negative impact on their survival. According to the national conference consensus recommendation, the priority for stage T1 HCC was abolished in April 2004. However, the priority for candidates with stage $2 \mathrm{HCC}$ has been maintained at $15 \%$ (equivalent to a MELD score of 24) (14). In April 2005, this priority was further reduced to a MELD score of 22 for stage T2 HCC.

Wide regional variations in DDLT for $\mathrm{HCC}$ are an important issue. Although we found no significant differences in waiting times, incidence rates, dropout rates and survival after LT in MELD 1 and 2 periods within each region, there was wide interregional variation. A higher incidence rate of DDLT for HCC was observed in region 3 and 10 in both periods. The average MELD scores for patients undergoing transplantation in regions 1,5 and 9 are probably higher than the priority MELD score for HCC in both periods, which may explain the lower DDLT incidence rate for $\mathrm{HCC}$ candidates in these regions. The waiting time was comparatively shorter in regions 3 and 10 than in regions 1,5 and 9 , probably because of the lower number of organs available for DDLT. The 12-month dropout rate in MELD periods 1 and 2 did not change significantly except in region 4, where there was a trend toward higher dropout in MELD 2. Since the majority of regions do not share organs except for those for status 1 candidates, even regional analysis most likely results in averaging and loss of information. In fact, a recent analysis of Scientific Registry of Transplant Recipient data revealed discrepancies in allocation from region to region and from donation service area to donation service area (15). These discrepancies could also be contributing to the variations in the incidence rates and dropout rates for $\mathrm{HCC}$ across regions.

Although the current liver allocation system for HCC is not perfect, LT for HCC has improved significantly under the MELD policy. Further enhancements to the process should take into account the following observations: (1) the natural history of HCC has not been well studied in patients with cirrhosis. Tumor burden and poorly differentiated histology are some of the factors that predict disease progression. Yet, according to the UNOS liver allocation policy, histologic findings are not a criterion for diagnosis of HCC before transplantation. (2) The priority for HCC candidates is still an evolving issue. In the past 3 years, priority scores for HCC candidates have been arbitrarily reduced 3 times. (3) There is considerable interregional variability in DDLT for HCC. On the basis of our findings, we would suggest that much can be done to improve DDLT for HCC. 
We recommend the following:

1 Available data indicate that the progression of stage T1T2 occurs slowly. Thus, it is appropriate to continue to list candidates with stage T1 HCC at their calculated MELD score.

2 Uniform guidelines should be developed for follow-up of these patients on the waitlist, because the progression from stage T2-stage T3/T 4 disease varies and depends on the total tumor burden, the rapid doubling time (less than 6 months), and the aggressiveness of the tumor ( $\alpha$-fetoprotein greater than $200 \mathrm{ng} / \mathrm{mL}$ )

3 Although there are insufficient data about the usefulness of palliative therapy for HCC, ablative therapies such as transarterial chemoembolization, radiofrequency ablation and percutaneous bland or ethanol ablation should be recommended in regions where the waiting time for these candidates is longer than 3 months. Prospective collection of this information by UNOS may help determine the usefulness of palliative therapy for HCC candidates awaiting $L T$ in the future.

4 A more uniform process and guidelines of allocation for HCC candidates should be developed at the regional level (e.g. interregional sharing of donor organs for patients with stage $\mathrm{T} 2 \mathrm{HCC}$ ).

\section{Acknowledgment}

These findings were presented as a late-breaking abstract at the annual meeting of the American Association for the Study of Liver Diseases held in Boston, Massachusetts, in October 2004. Editing was provided by the Section of Scientific Publications, Mayo Clinic. The authors would like to acknowledge Ms. Indu Lakhani, Department of Pediatrics, University of Michigan Hospital Systems for providing additional statistical support.

\section{References}

1. Mazzaferro V, Regalia E, Doci R et al. Liver transplantation for the treatment of small hepatocellular carcinomas in patients with cirrhosis. N Engl J Med 1996; 334: 693-699.

2. Otto G, Heuschen U, Hofmann WJ, Krumm G, Hinz U, Herfarth C. Survival and recurrence after liver transplantation versus liver resection for hepatocellular carcinoma: A retrospective analysis. Ann Surg 1998; 227: 424-432.

3. Bismuth $\mathrm{H}$, Chiche L. Comparison of hepatic resection and transplantation in the treatment of liver cancer. Semin Surg Oncol 1993; 9: 341-345.

4. Wiesner R, Edwards E, Freeman R et al. Model for end-stage liver disease (MELD) and allocation of donor livers. Gastroenterology 2003; 124: 91-96.

5. UNOS/OPTN. Modifications to policy 3.6.4.4 (Liver candidates with hepatocellular carcinoma). 3.6 Allocation of Livers. Accessed on 12/5/2005. http://www.unos.org/PoliciesandBylaws2/policies/ docs/policy $8 . d o c$

6. Sharma P, Balan V, Hernandez JL et al. Liver transplantation for hepatocellular carcinoma: The MELD impact. Liver Transpl 2004; 10: 36-41.

7. Yao FY, Bass NM, Ascher NL, Roberts JP. Liver transplantation for hepatocellular carcinoma: Lessons from the first year under the Model of End-Stage Liver Disease (MELD) organ allocation policy. Liver Transpl 2004; 10: 621-630.

8. Wiesner RH, Sorrell M, Villamil F. Report of the first International Liver Transplantation Society expert panel consensus conference on liver transplantation and hepatitis C. Liver Transpl 2003; 9: S19.

9. Wiesner RF, Freeman RB, Edwards E, Merion R, Harper A. The MELD/PELD liver allocation policy is associated with a reduction in waiting list mortality maintaining excellent post transplant patient and graft survival (Abstract). Gastroenterology 2003; 124: 708A.

10. Wiesner RH, Freeman RB, Mulligan DC. Liver transplantation for hepatocellular cancer: The impact of the MELD allocation policy. Gastroenterology 2004; 127: S261-S267.

11. Merion RM, Schaubel DE, Dykstra DM, Freeman RB, Port FK, Wolfe RA. The survival benefit of liver transplantation. Am J Transplant 2005; 5: 307-313.

12. Hayashi PH, Trotter JF, Forman L et al. Impact of pretransplant diagnosis of hepatocellular carcinoma on cadveric liver allocation in the era of MELD. Liver Transpl 2004; 10: 42-48.

13. Freeman RB. Liver allocation for HCC: A moving target. Liver Transpl 2004; 10: 49-51.

14. Olthoff KM, Brown RS, Jr, Delmonico FL et al. Summary report of a national conference: Evolving concepts in liver allocation in the MELD and PELD era. December 8, 2003, Washington, DC, USA. Liver Transpl 2004; 10: A6-22.

15. Hanto DW, Fishbein TM, Pinson CW et al. Liver and intestine transplantation: Summary analysis, 1994-2003. Am J Transplant 2005; 5: 916-933. 